\title{
Ser estudante de enfermagem em tempos de COVID-19
}

\author{
Being a nursing student in times of COVID-19 \\ Ser estudiante de enfermería en tiempos de COVID-19
}

\section{Cristina Lavareda Baixinho ${ }^{1}$ (D) \\ Óscar Ramos Ferreira ${ }^{1}$ (D)}

1. Escola Superior de Enfermagem de Lisboa, Centro de Investigação, Inovação e Desenvolvimento em Enfermagem de Lisboa. Lisboa, Portugal.
Autor correspondente:

Cristina Lavareda Baixinho.

Email: crbaixinho@esel.pt

Recebido em 22/12/2020.

Aprovado em 28/04/2021.

DOI:https://doi.org/10.1590/2177-9465-EAN-2020-0541

\section{Resumo}

Objetivo: Compreender como a pandemia afetou os estudantes de enfermagem nos contextos de Ensino Clínico. Método: Este é um estudo de abordagem qualitativa, exploratória, transversal e descritiva. Os participantes foram 5 enfermeiros recém-licenciados que realizaram o último estágio clínico da sua licenciatura durante a pandemia de COVID-19. A análise de conteúdo ocorreu por meio da técnica para a análise dos achados das narrativas. Resultados: Emergiram 5 categorias: Dificuldades sentidas na integração; Dificuldades na concretização do ensino clínico; Influência da pandemia nas expectativas dos estudantes ante o ensino clínico de integração à vida profissional; Receios, relacionadas com a COVID 19; e Sugestões para a formação dos licenciados em enfermagem, com vista a capacitá-los para a gestão de situações de pandemia. Conclusões e implicações para a prática: $O$ ensino clínico, cujo objetivo é atingir as competências exigidas para o exercício profissional, foi vivenciado com ansiedade, medo e pressão, porém com o suporte possível por parte dos supervisores clínicos, eles próprios também sob pressão, dado o aumento da sobrecarga de trabalho provocada pela pandemia. O estudo permite fazer recomendações para a educação e investigação em enfermagem.

Palavras-chave: Pandemias; Enfermagem; Estágio Clínico; Estudantes; Supervisão de Enfermagem.

\section{Abstract}

Aim: To understand how the pandemic has affected nursing students in clinical teaching contexts. Method: This was a qualitative exploratory, cross-sectional, and descriptive study. The participants were five newly graduated nurses who had completed the last clinical internship of their program during the COVID-19 pandemic. The narratives were analyzed using content analysis. Results: Five categories emerged: Difficulties felt in integration; difficulties in completing clinical internship; the influence of the pandemic on students' expectations regarding the clinical internship for integration into professional life; fears related to COVID-19, and suggestions for training nursing graduates to prepare them to manage pandemic situations. Conclusions and implications for practice: The clinical internship was experienced with anxiety, fear, and pressure to achieve the skills required for professional practice, with the possible support provided by clinical supervisors, who were also under pressure given the increased work overload caused by the pandemic. The study allows for recommendations for the area of nursing education and research.

Keywords: Pandemics; Nursing; Clinical Clerkship; Students; Nursing Supervisory.

\section{Resumen}

Objetivo: Comprender cómo afectó la pandemia a estudiantes de enfermería en el ámbito de la enseñanza clínica. Método: Estudio de abordaje cualitativo, exploratorio, transversal y descriptivo. Participaron 5 enfermeros recién graduados que realizaron la última práctica clínica de licenciatura durante la pandemia de COVID-19. Se aplicó análisis de contenido para estudiar los hallazgos narrados. Resultados: Surgieron 5 categorías: Dificultades percibidas en la integración; Dificultades en la concreción de la enseñanza clínica; Influencia de la pandemia en las expectativas estudiantiles respecto de la enseñanza clínica de integración a la vida profesional; Temores relacionados con la COVID-19; y Sugerencias para la formación de licenciados en enfermería con el objeto de capacitarlos para manejarse en situaciones de pandemia. Conclusiones e implicaciones para la práctica: La enseñanza clínica fue experimentada con ansiedad, miedo y presión para alcanzar las competencias exigidas para el ejercicio profesional., con el respaldo posible de los supervisores clínicos, ellos mismos también bajo presión dado el aumento de la sobrecarga de trabajo provocada por la pandemia. El estudio brinda recomendaciones sobre educación e investigación en enfermería.

Palabras clave: Pandemias; Enfermería; Prácticas Clínicas; Estudiantes; Supervisión de Enfermería 


\section{INTRODUÇÃO}

A Pandemia pelo SARS-CoV-2 tem imposto desafios complexos aos sistemas de saúde e à educação dos profissionais de saúde ${ }^{1-5}$, para garantir que a educação clínica se processe com a mesma qualidade, garantindo a segurança de doentes, profissionais e comunidade e, simultaneamente, possibilitando a aquisição de conhecimentos e o desenvolvimento de competências.

Para responder aos desafios que a pandemia colocou, as instituições de ensino superior responsáveis pela formação de profissionais de enfermagem têm ajustado o plano curricular, optando por uma modalidade de B-learning para as aulas teóricas, teórico-práticas e de práticas laboratoriais. Os estudantes, enquanto elementos ativos no processo de ensino-aprendizagem, alternam-se entre aulas de matriz teórica e tipologias de ensino de natureza prática em serviços que prestam cuidados de saúde, para adquirirem as competências necessárias para uma prática clínica de qualidade e que garanta a segurança dos cidadãos ${ }^{4,5}$.

A aprendizagem clínica é um elemento central da educação em enfermagem, reconhecida como um dos fatores mais importantes para o desenvolvimento de competências ${ }^{6}$. Ante a pandemia, o Estágio Clínico (EC), tanto em contexto hospitalar como de cuidados de saúde primários, tem mantido o regime presencial em estágio, embora adaptado às circunstâncias impostas pela situação. Quanto aos resultados de aprendizagem pretendidos com esse tipo de ensino, eles não sofreram alterações, apesar das difíceis circunstâncias em que se processam.

Em Portugal, o Curso de Licenciatura em Enfermagem (CLE) comtempla a realização de EC em contextos de cuidados específicos e com clientes que experienciam diferentes transições de desenvolvimento, saúde-doença, situacionais e organizacionais. O estágio constitui-se como momento privilegiado para a integração teórico-prática do conhecimento ${ }^{6}$, pelo confronto entre o saber acadêmico e a sua integração nas situações de práxis clínica dos profissionais. Sendo o EC um processo por excelência para a aprendizagem e consequente aquisição e desenvolvimento de competências, tem de possibilitar o envolvimento e a motivação dos estudantes ${ }^{6}$ e, perante as vicissitudes supracitadas, estabelecer um conjunto de estratégias para melhorar a qualidade da educação clínica ${ }^{7,8}$.

É certo que a pandemia COVID-19 alterou o nosso cotidiano e também a educação em enfermagem ${ }^{1,2}$, levando-a para muito além daquilo que é o ensino presencial, ou seja, para uma realidade, também ela, marcada pela educação a distância. Numa primeira fase, ao ser decretado um período de confinamento obrigatório, os estudantes tiveram as suas experiências clínicas suspensas, tal como em outros países ${ }^{2}$. Após três meses de interrupção, regressaram aos contextos clínicos, sendo que alguns estudantes ficaram na linha da frente, testemunhando os efeitos da doença nas diferentes transições de vida da população ${ }^{1,2}$, especialmente da mais vulnerável para este tipo de infeção. Em algumas situações, com o surgimento da pandemia COVID 19, os estudantes foram colocados numa posição dilemática, na medida em que tiveram de escolher entre a sua segurança e a da sua família e o prosseguimento dos seus estudos e consequente carreira profissional ${ }^{1}$, sobretudo os estudantes com doenças crônicas ou oncológicas.
Em ambos os casos, o medo, a incerteza e a preocupação têm afetado os estudantes ${ }^{1}$. O medo de contágio individual e a incerteza quanto à manifestação de sinais, de sintomas e ao prognóstico da doença, aliados ao receio de que a suspensão do estágio ou o contágio durante a sua realização interferissem nos planos de finalizar a licenciatura e de ingressar no mercado de trabalho, produziram um estado de maior ansiedade e estresse ${ }^{1}$.

Os autores observaram que, além dos riscos de contaminação, os estudantes estão sujeitos também a alterações psicoemocionais ${ }^{5}$ decorrentes de toda essa problemática, até pela dualidade entre o medo e o desejo de cumprir a missão social da profissão que escolheram. Estudos recentes reportam que os estudantes e estagiários de enfermagem e de medicina dos Estados Unidos e Europa têm vivenciado sentimentos de ansiedade e vulnerabilidade que competem internamente com o desejo e o compromisso de cuidar de pacientes com a Covid-193,4.

A identidade profissional é importante para os futuros enfermeiros. Os estudantes sentem uma recompensa intrínseca ao assumir o seu papel em contexto clínico, até porque para alguns ser enfermeiro está ligado à noção de enfermagem como uma vocação, e muitos estudantes descrevem isso como uma motivação fundamental na sua escolha de carreira ${ }^{3}$ e, em situação de crise, sentem o 'apelo vocacional'.

Alguns peritos advogam que, apesar da existência de determinados aspectos negativos, a educação durante as pandemias também pode ser vista como uma oportunidade para que os estudantes desenvolvam ou fortaleçam competências já em processo de construção, desde os anos iniciais da graduação². Salientam esses entendidos que, no Ensino Superior, é fundamental não apenas fornecer orientação e treino aos estudantes em relação à sua prática de enfermagem, mas também prover apoio em tempos de crise ${ }^{1}$ como este que estamos vivendo.

A pandemia provocada pelo SARS-CoV-2 e a necessidade concomitante de aumentar o número de enfermeiros para the fazer face proporcionaram àqueles cuja opção foi realizar o EC a oportunidade de fazer algo que normalmente não teriam feito e de consequentemente obterem outras aprendizagens ${ }^{3}$.

Partindo da premissa de que os desafios atuais aos sistemas de saúde e à organização dos cuidados, em virtude da COVID-19, para garantir a segurança dos doentes, dos profissionais e da comunidade, têm imposto alterações ao processo de ensinoaprendizagem dos estudantes que estão em estágio clínico nos diferentes contextos de saúde, é objetivo deste estudo compreender como a pandemia afetou os estudantes de enfermagem nos contextos de Estágio.

\section{MÉTODO}

A natureza do fenômeno investigado e os poucos estudos sobre o tema orientaram o desenho desta pesquisa exploratória, de abordagem qualitativa, transversal e descritiva. A investigação foi realizada no hospital parceiro da Escola Superior de Enfermagem de Lisboa, no âmbito do projeto transição segura, o qual acolhe estudantes de enfermagem para a realização do último EC do CLE de integração à vida profissional.

A partir da questão central "Como a pandemia influenciou o Ensino Clínico?", solicitou-se aos participantes uma narrativa 
escrita. Para orientar a narrativa, redigiu-se um roteiro com questõesestímulo, com a finalidade de um maior controle sobre o que se pretendia compreender e simultaneamente possibilitar espaço para uma reflexão livre e espontânea dos participantes sobre o tema ${ }^{9}$.

A narrativa é uma técnica importante para a coleta de dados na investigação qualitativa porque permite estabelecer objetivos e formular opções para a ação, baseada na prática, teoria e investigação, para uma intervenção caracterizada por um nível mais elevado de saberes, mobilizando para tal a experiência vivenciada ${ }^{10}$.

Os participantes deste estudo foram cinco estudantes $(E)$ finalistas do CLE os quais realizaram o último EC em ambiente hospitalar. Cada um deles foi contatado por um dos investigadores, para solicitar a sua participação e garantir que seriam respeitados os critérios de inclusão definidos: estudante finalista, estar em EC num serviço com alterações de organização decorrente do Plano Nacional de Preparação e Resposta para a doença por novo coronavírus (COVID 19) e das orientações internas das instituições para a organização dos cuidados, e aceder participar livre e esclarecidamente do estudo.

A opção pela seleção dos participantes possibilitou a focalização na discussão do fenômeno em estudo, já que os participantes tinham em comum uma característica relevante ante o tema em discussão - realizar EC em período de COVID 19, em instituição hospitalar.

A colheita de dados foi efetuada na segunda semana de agosto, após a saída dos resultados da avaliação do EC, de forma a minimizar o efeito de respostas 'socialmente desejáveis'.

A análise de conteúdo ${ }^{11}$ foi feita por meio da técnica utilizada para descrever e interpretar o discurso dos participantes. $O$ protocolo seguido incluiu a preparação das informações, a transformação do conteúdo em unidades, a categorização, a descrição e a interpretação9,11. Após a leitura flutuante, iniciouse a exploração do conteúdo, com seleção das unidades de codificação (recorte, enumeração, escolha de categorias) e categorização, mantendo um diálogo contínuo e bidirecional entre a teoria e os dados ${ }^{11}$.

No processo de análise, valorizaram-se domínios como: 0 contexto, as pessoas, os processos e o tempo em que a ação decorreu, de forma a criarem-se condições para que a reflexão sistemática ocorresse, considerando-se a diversidade e a singularidade da experiência ${ }^{10}$ destes estudantes num contexto clínico em mudança. Na definição das categorias, obedeceu-se às regras da exaustividade, representatividade, homogeneidade, pertinência e exclusividade ${ }^{9,11}$. A análise efetuada pelos investigadores foi devolvida aos participantes para validação.

Este estudo foi autorizado por uma Comissão de Ética (Parecer 09/HVFX/2019). As instituições envolvidas têm um protocolo de parceria formalizado que autoriza a realização de investigação. Foram respeitados todos os pressupostos éticos e formais, desde a autorização para integrarem o estudo, com assinatura do termo de consentimento de participação livre $e$ esclarecida, até as questões éticas inerentes ao desenvolvimento da investigação. Foi ainda assegurado a todos os participantes o anonimato e a confidencialidade dos dados.

\section{RESULTADOS}

A amostra foi constituída por cinco (5) estudantes (E) finalistas que realizaram o último Ensino Clínico do Curso de Licenciatura de Enfermagem da Escola Superior de Enfermagem de Lisboa, nos serviços de um hospital da grande Lisboa, no decurso da primeira onda da pandemia de COVID-19 que assolou o país. Esse ensino clínico terminou em julho de 2020. A média de idade dos enfermeiros foi de 21,8 anos de idade, sendo que $80 \%$ deles tinham 22 anos.

Os participantes realizaram EC nos serviços de Urgência, Cuidados Intensivos, Especialidades Cirúrgicas e Especialidades Médicas.

Da análise do discurso dos participantes, emergiram 5 categorias: Dificuldades sentidas na integração associadas à pandemia; Dificuldades na concretização do EC associadas à pandemia; Influência da pandemia nas expectativas dos estudantes ante o EC de integração à vida profissional; Receios relacionadas com a COVID 19 durante o EC; e Sugestões para formação dos licenciados em enfermagem, com vista a capacitá-los para a gestão de situações de pandemia. Na Tabela 1, apresentam-se as categorias e respetivas frequências de registro presentes no conteúdo analisado. A identificação de 101 unidades reforça a riqueza do discurso dos estudantes, possibilitando a interpretação e inferência do conteúdo dos achados a partir de cada unidade analisada e devidamente classificada, observando os requisitos de exaustividade, exclusividade, homogeneidade, 'confiabilidade e validade.

\section{Dificuldades sentidas na integração associadas à pandemia}

As dificuldades sentidas pelos estudantes na sua integração em EC associadas à pandemia relacionaram-se com a utilização de máscara que, segundo estes, "prejudica a comunicação" (E1), com a necessidade de conviver com profissionais exercendo na área COVID e doentes COVID+ mantendo o distanciamento social, e com o fato de ter de se preparar para realizar EC noutro local que não aquele onde tinham iniciado o EC (E1, E2).

Tabela 1. Categorias e Frequência de Unidades de Registro por Categoria. Lisboa, 2020

\section{Categorias}

\begin{tabular}{lc}
\hline Dificuldades sentidas na integração & $\mathbf{2 7}$ \\
$\begin{array}{l}\text { Dificuldades na concretização do EC } \\
\text { Influência da pandemia nas expectativas dos }\end{array}$ & 21 \\
$\begin{array}{l}\text { estudantes ante o EC de integração à vida } \\
\text { profissional }\end{array}$ & 19 \\
$\begin{array}{l}\text { Receios relacionados com a COVID 19 } \\
\begin{array}{l}\text { Sugestões para formação dos licenciados } \\
\text { em enfermagem, com vista a capacitá-los } \\
\text { para a gestão de situações de pandemia. }\end{array}\end{array}$ \\
\begin{tabular}{l} 
Total \\
\hline
\end{tabular} & 11 \\
\hline
\end{tabular}


Também a interrupção de três (3) meses, por confinamento obrigatório, foi sentida como uma dificuldade, dado haver "receio de regressar ao contexto de ensino clínico" (E2), tendose "sentido um retrocesso" (E4) na capacidade de construir raciocínios clínicos e "dificuldades na destreza" (E4) quando isso aconteceu. A ausência dos locais de EC e o confinamento em casa durante tanto tempo impediram que os estudantes desenvolvessem o raciocínio clínico que a prática clínica diária e sistemática permite, bem como interrompeu o desenvolvimento de competências instrumentais adquiridas.

Outra das dificuldades de integração que sentiram relacionouse com a sobrecarga que a pandemia acarretou, tanto para os serviços quanto para os profissionais (E5), e muitos deles, para além das funções inerentes ao exercício profissional nos serviços, assumiam ainda a função de orientadores clínicos. Tal sobrecarga pode ter tirado a disponibilidade para uma integração adequada dos estudantes.

Um dos estudantes (E3) que realizou EC num serviço de cirurgia, ao contrário dos outros, referiu que não sentiu dificuldades associadas à pandemia. A justificativa para a ausência de dificuldades nesse serviço relacionou-se com o fato de, apesar de ele possuir plano de contingência e ter sofrido alterações decorrentes da organização interna dos serviços de internamento, não ter recebido nenhum doente com infeção ativa no período de EC da aluna. Esse afastamento da linha de frente (serviços de urgência, medicina e cuidados intensivos) em que o combate à pandemia era uma constante parece ter influenciado a integração e a disponibilidade dos enfermeiros para a integração da estudante. Outra das razões pode estar relacionada com o fato de essa estudante já conhecer o serviço e de nele ter sido bem integrada previamente à instituição do confinamento a que a população portuguesa foi então obrigada.

\section{Dificuldades na concretização do EC associadas à pandemia}

Os estudantes entenderam que a redução do tempo de ensino clínico "Redução das horas de contato com doentes e equipe dos serviços, ou seja, do tempo de estágio" (E1) foi uma das principais dificuldades na concretização do EC associado à pandemia, o que levou a que tivessem de "adquirir o máximo conhecimento teórico-prático num período de 6 semanas" (E2). Mas também as dificuldades em contactarem os familiares dos doentes (E4) e em gerirem o seu tempo pela quantidade insuficiente dos serviços de profissionais de enfermagem, situação que já se manifestava antes da pandemia e que esta acabou por agravar (E4), com a consequente diminuição da disponibilidade dos enfermeiros presentes para acolherem, orientarem, supervisarem e avaliarem convenientemente os estudantes.

O confinamento obrigatório, pela "perda de ritmo" (E5) a que obrigou, acabaria também por sujeitar os estudantes a "um maior esforço para conseguir recuperar o tempo perdido" (E5) e consequentemente concretizar o EC de forma satisfatória. A estudante que realizou EC nas especialidades cirúrgicas (E3) continuou a referir que a pandemia "não influenciou" a concretização do EC, o que corrobora o que afirmamos anteriormente, até porque, se os enfermeiros do serviço não estivessem na linha de frente do combate à pandemia, decerto teriam mais disponibilidade para acompanharem convenientemente essa estudante durante a sua estadia no serviço.

\section{Influência da pandemia nas expetativas dos estudantes ante o EC de integração à vida profissional}

A pandemia parece ter baixado as expetativas dos estudantes ante o EC, por receio de insucesso. Fracasso relacionado com a possibilidade de adoecerem pelo contato com infectados e por exaustão. Um estudante referiu o quão prejudicial seria, para a prestação de cuidados, o medo que a pandemia trouxe de ter contato com o outro: "Houve sempre um certo receio no contacto com o outro, algo que é prejudicial para a prestação de cuidados" (E1). A baixa de expetativas relacionou-se também com a possibilidade de o EC ser bem mais exigente que o inicialmente planejado, pelo aumento da pressão para desenvolver as competências previstas com a consequente exaustão física e psicológica, dada a redução do tempo de EC para seis (6) semanas, conforme afirmaram dois dos respondentes: "expectativas de um nível de dificuldade mais elevado e de aumento do nível de exaustão física e psicológica. (...), visto que teria de adquirir os mesmos conhecimentos em 6 semanas, ao invés de 4 meses" (E2); "Gerou uma maior pressão, (...) pois não permitiu que certas competências fossem desenvolvidas ou que tivessem de ser desenvolvidas mais depressa" (E4).

De fato, as expetativas parecem ter sido tão reduzidas quanto à possibilidade de desenvolverem as competências previstas para o EC que uma estudante se mostrou admirada com o sucesso obtido, pelo que referiu que "a conclusão (...) com êxito face à situação em que decorreu deixou-me mais confiante e com mais segurança" (E5). Mais uma vez, e curiosamente, a estudante que realizou EC nas especialidades cirúrgicas revelou que a pandemia "não influenciou" (E3) as expetativas que tinha ante o EC.

\section{Principais receios relacionadas com a COVID 19 durante o EC}

Os principais receios dos estudantes referentes à COVID-19 durante o EC relacionaram-se, por ordem decrescente, com a possibilidade de serem infectados: "este estágio não foi fácil, a palavra que o define é medo, primeiro foi o medo de não irmos para estágio e, depois de lá estarmos, o medo de sermos contagiados, parece estranho quando dizem que os jovens não têm formas graves de doença, mas, no serviço ao lado do meu, estavam alguns jovens" (E3); e de transmitirem COVID-19 a familiare e amigos: "assim que chegava a casa tirava a roupa e tomava banho, não sabia o que trazia do hospital para casa" (E1); de não concluírem o curso: "quando nos disseram que os critérios de escolha do estágio seriam os mesmos, pensei que, por ter uma média baixa, tão depressa não conseguiria estagiar, o que seria difícil no meu caso, preciso mesmo de começar a trabalhar" (E4); e de, enquanto estudantes, voltarem a prestar cuidados após um interregno de três (3) meses: "comecei o estágio, estive uma semana num serviço e, passados 3 meses, volto para outro completamente diferente, não estava preparada, 
perdi algumas coisas neste tempo (...), não tanto conhecimento, mas destreza e dificuldade em avançar” (E2).

\section{Sugestões para a formação dos licenciados em enfermagem com vista a capacitá-los para a gestão de situações de pandemia}

Os quatro estudantes que referiram que a escola os preparou com formação para realização de $\mathrm{EC}$ em contextos de risco de contágio sugeriram que seria importante: "reforçar a importância do distanciamento social com os doentes (...) e com a equipe de saúde" (E1) e fazer "formação nesse âmbito" (E3), realizando cursos, discutindo "conhecimento com os colegas e restante equipe" (E4), cursos esses que poderiam ser "online", nomeadamente "sobre a pandemia" (E5). Para além disso, esse respondente $(E 5)$ sugere que seria importante um "acréscimo de documentação referente ao controle de infeção e sobre a pandemia" (E5).

O participante que referiu não ter sido preparado para a realização de EC em contextos de risco de contágio sugeriu "disponibilização de apoio psicológico" (E2) pela escola, bem como o investimento desta "em formações específicas sobre a pandemia" (E2), o que está alinhado com o sugerido pelos outros participantes.

\section{DISCUSSÃO}

Os resultados desta pesquisa reforçam a afirmação de que a pandemia alterou o paradigma da educação clínica ${ }^{12}$. Alguns autores consideram mesmo que a enfermagem e a educação em enfermagem estão entrando numa nova era ${ }^{1}$, com necessidade de explorar novas estratégias pedagógicas e avaliar a sua efetividade.

Os achados desta pesquisa apontam que os estudantes vivenciaram o ensino clínico com medo de serem infectados e de transmitirem a COVID-19 a familiares e amigos e de voltarem a ter contato com pessoas e prestarem cuidados, o que é corroborado por estudos recentes sobre o tema que concluem que a ansiedade entre estudantes de enfermagem é elevada durante a pandemia de Covid-191,13-16. Um estudo que decorreu na Espanha e que avaliou os níveis de estresse em 3 momentos (um antes e dois durante 0 confinamento) concluiu que estes aumentaram substancialmente durante o período de isolamento social ${ }^{16}$.

Os motivos que levaram os estudantes a ficarem ansiosos estão relacionados com o risco de serem infectados. Um estudo refere que $68,1 \%$ dos estudantes manifestam essa preocupação ${ }^{14}$, com a incerteza e os problemas econômicos ${ }^{14,16}$, com os problemas familiares ${ }^{16}$; com o medo da infeção no seio da sua família ${ }^{14}$, com a necessidade de apoiar e cuidar dos descendentes ${ }^{14} \mathrm{e}$ com a falta de recursos para lidar com os desafios das novas modalidades pedagógicas ${ }^{13}$.

Outro sintoma preocupante resultante da ansiedade relaciona-se com a prevalência da má qualidade do sono e consequente mudança do seu padrão entre os estudantes de enfermagem, a qual tem sido associada a desempenho reduzido, mudanças comportamentais, mudanças na dieta e até mesmo a comportamento agressivo ${ }^{15}$. As restrições às diferentes atividades de vida podem resultar em mudanças no estilo de vida que afetam a qualidade do sono ${ }^{15}$, o que, aliado ao horário rotativo a que o ensino clínico obriga, pode contribuir para o risco de exaustão.

Em relação às categorias que emergiram e que estão relacionadas com as dificuldades na integração e na concretização do EC, corroboramos a opinião dos médicos que referem que a manutenção da educação clínica, apesar dos riscos, traz benefícios para os doentes e para os sistemas de saúde, porque a participação dos estudantes reforça valores importantes, como o altruísmo, o atendimento em momentos de crise e a solidariedade na profissão ${ }^{17}$. Porém é com alguma preocupação que analisamos e discutimos o papel dos orientadores clínicos e docentes nesse processo.

Para que as experiências clínicas possibilitem aprendizagens duradouras e positivas, há que repensar a forma como é feita a integração nos serviços e o papel que o orientador clínico tem nesse processo, especialmente em períodos de maior exigência e complexidade na prestação de cuidados. Os achados desta pesquisa reportam dificuldades nesse nível, não só pelo receio dos estudantes e pelo afastamento temporário dos contextos, o que trouxe maiores inseguranças quanto à habilidade prática, destreza manual e ao raciocínio clínico em enfermagem, mas também porque a sobrecarga de trabalho sobre os profissionais de enfermagem diminuiu a sua disponibilidade para uma integração adequada dos estudantes e um apoio efetivo ao longo de todo o processo educativo.

As dificuldades na integração e no desenrolar do EC afetam o processo de ensino-aprendizagem. A inserção do estudante no mundo real do trabalho, tutorado pela figura concreta do profissional enfermeiro, mediado pela organização didático-pedagógica do docente, tem por objetivos realizar intervenções reais nos processos de trabalho e promover a formação de profissionais capazes de fazer com que a práxis (articulação teoria-prática) subsidie reflexões coletivas, resultando em modificações nas situações reais de saúde ${ }^{18,19}$.

A tutoria desempenhada pelos enfermeiros clínicos tem sido reconhecida, na literatura internacional, como uma estratégia para maximizar os benefícios da educação em enfermagem em termos de aquisição de conhecimentos e habilidades, segurança e socialização profissional ${ }^{20}$, o que fica comprometido a partir do momento em que a disponibilidade desses profissionais diminui, fruto das exigências do contexto e da complexidade a que o momento pandêmico obriga.

A supervisão de enfermagem durante o EC não pode ser desvalorizada, mesmo numa situação complexa como a da infeção pelo SARS-CoV-2, pelo risco de comprometimento do processo de socialização, ensino e avaliação dos estudantes, dificultando a integração da teoria na prática ${ }^{18,20}$, e a aquisição de novo conhecimento, habilidades e atitudes para desenvolver a competência profissional ${ }^{19}$. Essa preocupação está presente no discurso escrito pelos participantes, que manifestaram a preocupação, no início e durante o EC, de que a diminuição do número de horas para a sua realização pudesse afetar o desenvolvimento de capacidades e competências para o exercício autônomo da profissão. Sobressai ainda, na análise do discurso, a apreensão em relação à capacidade de mobilizar, de forma consciente e sistematizada, as aprendizagens cognitivas, psicomotoras e atitudinais na tomada de decisão na clínica ${ }^{6,18-20}$. 
Estudos futuros devem explorar se a experiência de EC em tempos COVID influenciou a integração na vida profissional e se esta se constituiu como um evento traumático. Como observa outro estudo, o impacto da pandemia sobre estudantes e profissionais, sobretudo naqueles que são mais vulneráveis, exigirá cuidados e atenção especiais para garantir que sejam bem-sucedidos ${ }^{1}$. Decerto que, se a integração ocorrer no serviço onde o último EC foi desenvolvido, o impacto será nulo ou francamente menor. Mas cabe a pesquisas futuras confirmar ou refutar essa hipótese.

Por outro lado, o receio e a ansiedade, diante de uma situação nova e complexa, com riscos para a própria segurança e de seus familiares, alertam para a necessidade de preparar os futuros profissionais para experiências traumáticas ou eventos adversos $^{1}$ e de apoiá-los psicoemocionalmente. Essa ajuda psicoemocional pode ser conseguida por meio de cuidados de saúde mental, em que se inclui o apoio psicológico, o que já foi evidenciado em crises epidemiológicas anteriores, sendo agora reforçada diante da atual pandemia ${ }^{21}$.

Uma pesquisa realizada no Japão, com enfoque nas experiências, no sentimento de pertencimento e nos processos de tomada de decisão, desvela que, embora a COVID-19 tenha influenciado fortemente a compreensão, o feedback e os padrões de vida dos participantes, o sentimento de pertencimento e a tomada de decisão permaneceram inalterados e, em algumas situações, saíram reforçados pela certeza sobre a escolha profissional e a sua missão social ${ }^{22}$.

Cremos que o desenvolvimento da identidade profissional e definição da missão social dos educadores de enfermagem têm um papel crítico para preparar os estudantes para trabalharem em ambientes de saúde complexos e em constante mudança ${ }^{23}$.

Não sendo âmbito deste estudo, consideramos que é importante explorar as motivações dos estudantes que optaram por não realizar EC em tempos de pandemia, bem como as consequências de tal decisão. As motivações por trás de tal decisão podem estar relacionadas com o facto de esses estudantes viverem com pessoas de um grupo de risco; ou eles próprios pertencerem também a um dos grupos de risco; ou por se sentirem despreparados para os desafios colocados pela pandemia. Uma pesquisa por nós identificada refere que essa opção colocou os estudantes em uma situação difícil, e muitos deles com sentimentos de culpa ${ }^{24}$, o que pode influenciar as experiências clínicas futuras.

A sugestão de um dos participantes foi a de que os estudantes pudessem ter apoio psicológico, o que vai ao encontro da bibliografia por nós consultada. Esse apoio antes e durante o EC pode auxiliar os estagiários a lidarem com o estresse e, com isso, permitir que eles possam desenvolver também habilidades para se protegerem a si, a suas famílias e à comunidade durante infecções epidêmicas ${ }^{14}$.

A educação na enfermagem e nas outras disciplinas da saúde deve promover o pensamento crítico, a reflexão e a tomada de decisão segura, garantindo aos clientes dos cuidados de saúde o melhor cuidado ao menor custo ${ }^{19}$. A ideia contida nessa afirmação é uma preocupação internacional e um desafio para a educação, uma vez que para isso é necessário que os estudantes possuam conhecimentos, atitudes e competências para uma prática clínica segura e baseada em evidência. Estudos futuros devem explorar se ter sido estudante de enfermagem em tempos de COVID-19 tornou-os melhores enfermeiros ${ }^{24} \mathrm{e}$ profissionais mais capazes de enfrentarem situações adversas e complexas. A recomendação de um participante foi a de que as instituições de ensino desenvolvam formação nesse âmbito. Mesmo após o término desta pandemia, recomenda-se a criação de espaços de debate/discussão de planos de contingência e debate com base em estudos de caso sobre a COVID-19 nos currículos de enfermagem ${ }^{25}$, aproveitando as potencialidades da prática simulada de alta fidelidade para o efeito ${ }^{26}$.

\section{CONSIDERAÇÕES FINAIS}

A análise de conteúdo das narrativas dos cinco participantes permite compreender que vivenciaram o estágio clínico com um misto de sentimentos: receio de insucesso, medo de contágio, motivação para terminar o seu plano curricular e preocupação com o desenvolvimento de competências que os preparasse para o exercício de funções. Destacam-se dificuldades na integração e concretização do EC relacionadas com a interrupção, por um período de 3 meses, e pelo encurtamento do número de horas. Os planos de contingência, as medidas de controle de infeção e a evolução da infeção pelo SARS-CoV-2 condicionaram a integração, a comunicação e a disponibilidade dos supervisores clínicos para a orientação dos estudantes.

Os achados abrem a porta para a discussão pública sobre as oportunidades de aprendizagem e as dificuldades que os estudantes têm em ensino clínico. A pandemia, as mudanças organizacionais e o acréscimo de volume de trabalho afetaram o tempo e a disponibilidade dos orientadores clínicos, o que influenciou a aprendizagem e a experiência clínica do estudante que está vulnerável diante da dualidade entre a noção de obrigação de ajudar e os medos associados.

Essa discussão deve-se estender ao papel das instituições de ensino e dos docentes, para garantir a qualidade da educação em enfermagem, e tem implicações para a prática clínica e pedagógica dos enfermeiros supervisores. Por isso, recomenda-se que, nos cursos de formação em supervisão de enfermagem, que sejam abordadas as questões referentes ao papel e às atividades do supervisor clínico em tempo de pandemias como facilitador da integração dos estudantes e como suporte à sua aprendizagem.

O tamanho da amostra que não permitiu a saturação dos achados constitui-se como a principal limitação deste estudo. Pesquisas futuras com amostras maiores e, eventualmente, guiadas pelas categoriais que emergiram deste estudo, devem aprofundar os achados deste estudo.

\section{FINANCIAMENTO}

O projeto "Transição Segura" é financiado pelo Centro de Investigação, Inovação e Desenvolvimento em Enfermagem de Lisboa (CIDNUR).

\section{CONTRIBUIÇÕES DOS AUTORES}

Desenho do estudo. Cristina Lavareda Baixinho. Óscar Ramos Ferreira. 
Coleta ou produção dos dados. Cristina Lavareda Baixinho. Óscar Ramos Ferreira.

Análise de dados. Cristina Lavareda Baixinho. Óscar Ramos Ferreira.

Interpretação dos resultados. Cristina Lavareda Baixinho. Óscar Ramos Ferreira.

Redação e revisão crítica do manuscrito. Cristina Lavareda Baixinho. Óscar Ramos Ferreira.

Aprovação da versão final do artigo. Cristina Lavareda Baixinho. Óscar Ramos Ferreira.

Responsabilidade por todos os aspectos do conteúdo e a integridade do artigo publicado. Cristina Lavareda Baixinho. Óscar Ramos Ferreira.

\section{EDITOR ASSOCIADO}

Antonio Jose Almeida Filho (1)

\section{EDITOR CIENTÍFICO}

Ivone Evangelista Cabral (1)

\section{REFERÊNCIAS}

1. Fowler K, Wholeben M. COVID-19: outcomes for trauma-impacted nurses and nursing students. Nurse Educ Today. 2020 out;93:104525. http://dx.doi.org/10.1016/j.nedt.2020.104525. PMid:32659536.

2. Franzoi MAH, Cauduro FLF. Participation of nursing students in the Covid-19 pandemic. Cogitare Enferm. 2020;25:e73491. http://dx.doi. org/10.5380/ce.v25i0.73491.

3. Swift A, Banks L, Baleswaran A, Cooke N, Little C, McGrath L et al. COVID-19 and student nurses: a view from England. J Clin Nurs. 2020;29(17-18):3111-4. http://dx.doi.org/10.1111/jocn.15298. PMid:32298512.

4. Gallagher TH, Schleyer AM. "We signed up for this!": student and trainee responses to the Covid-19 pandemic. N Engl J Med. 2020;382(25):e96. http://dx.doi.org/10.1056/NEJMp2005234. PMid:32268020.

5. Miller DG, Pierson L, Doernberg S. The role of medical students during the COVID-19 pandemic. Ann Intern Med. 2020;173(2):145-6. http:// dx.doi.org/10.7326/M20-1281. PMid:32259194.

6. Jamshidi N, Molazem Z, Sharif F, Torabizadeh C, Najafi Kalyani M. The challenges of nursing students in the clinical learning environment: a qualitative study. ScientificWorldJournal. 2016;2016:1846178. http:// dx.doi.org/10.1155/2016/1846178.

7. Albarqouni L, Hoffmann T, Straus S, Olsen NR, Young T, llic D et al. Core competencies in evidence-based practice for health professionals: consensus statement based on a systematic review and delphi survey. JAMA Netw Open. 2018;20181(2):e180281. http://dx.doi.org/10.1001/ jamanetworkopen.2018.0281. PMid:30646073.

8. Shadadi H, Sheyback M, Balouchi A, Shoorvazi M. The barriers of clinical education in nursing: a systematic review. Biomed Res. 2018;29(19):361623. http://dx.doi.org/10.4066/biomedicalresearch.29-18-1064.

9. Minayo C, Costa AP. Técnicas que fazem uso da palavra, do olhar e da empatia. Oliveira de Azeméis: Ludomédia; 2019.

10. Silva IB, Amendoeira J. The use of narrative in the paradigm of qualitative research. Rev UIIPS [Internet]. 2018; [citado 2020 dez 22];2(VI):29-40. Disponível em: http://ojs.ipsantarem.pt/index.php/REVUIIPS

11. Bardin L. Análise de conteúdo. Lisboa: Edições 70; 2011.
12. Hilburg R, Patel N, Ambruso S, Biewald MA, Farouk SS. Medical education during the coronavirus disease-2019 pandemic: learning from a distance. Adv Chronic Kidney Dis. 2020 jun 23;27(5):412-7. http://dx.doi.org/10.1053/j.ackd.2020.05.017. PMid:33308507.

13. Savitsky B, Findling Y, Ereli A, Hendel T. Anxiety and coping strategies among nursing students during the covid-19 pandemic. Nurse Educ Pract. 2020 jul; 46:102809. http://dx.doi.org/10.1016/j.nepr.2020.102809. PMid:32679465.

14. Aslan H, Pekince H. Nursing students' views on the COVID-19 pandemic and their percieved stress levels. Perspect Psychiatr Care. 2021 abr;57(2):695-701. http://dx.doi.org/10.1111/ppc.12597. PMid:32808314.

15. Romero-Blanco C, Rodríguez-Almagro J, Onieva-Zafra MD, ParraFernández ML, Prado-Laguna MDC, Hernández-Martínez A. Sleep pattern changes in nursing students during the COVID-19 lockdown. Int $J$ Environ Res Public Health. 2020;17(14):5222. http://dx.doi.org/10.3390/ ijerph17145222. PMid:32698343.

16. Gallego-Gómez Jl, Campillo-Cano M, Carrión-Martínez A, Balanza S, Rodríguez-González-Moro MT, Simonelli-Muñoz AJ et al. The COVID-19 pandemic and its impact on homebound nursing students. Int J Environ Res Public Health. 2020;17(20):7383. http://dx.doi.org/10.3390/ ijerph17207383. PMid:33050435.

17. Miller DG, Pierson L, Doernberg S. The role of medical students during the COVID-19 pandemic. Ann Intern Med. 2020;173(2):145-6. http:// dx.doi.org/10.7326/M20-1281. PMid:32259194.

18. Esteves LSF, Cunha ICKO, Bohomol E, Negri EC. Supervised internship in undergraduate education in nursing: integrative review. Rev Bras Enferm. 2018;71(Supl. 4):1740-50. http://dx.doi.org/10.1590/00347167-2017-0340. PMid:30088648.

19. Baixinho CL, Ferreira Ó, Cardoso M, Nascimento P, Pedrosa R, Gonçalves P. Autopercepção dos estudantes sobre a participação em atividades extracurriculares de transferência de conhecimento: 0 exemplo da Transição Segura. NTQR. 2020;2:588-601. http://dx.doi. org/10.36367/ntqr.2.2020.588-601.

20. Iglesias-Parra MR, García-Mayor S, Kaknani-Uttumchandani S, LeónCampos A, García-Guerrero A, Morales-Asencio JM. Nursing students' and tutors' satisfaction with a new clinical competency system based on the Nursing Interventions Classification. Int J Nurs Knowl. 2016;27(4):193200. http://dx.doi.org/10.1111/2047-3095.12102. PMid:26033284.

21. Moreira WC, Sousa AR, Nóbrega MPSS. Adoecimento mental na população em geral e em profissionais de saúde durante a Covid-19: scoping review. Texto Contexto Enferm. 2020. In press. http://dx.doi. org/10.1590/SciELOPreprints.689.

22. Santos LMD. The relationship between the COVID-19 pandemic and nursing students' sense of belonging: the experiences and nursing education management of pre-service nursing professionals. Int $J$ Environ Res Public Health. 2020 ago 12;17(16):5848. http://dx.doi. org/10.3390/ijerph17165848. PMid:32806697.

23. Taylor R, Thomas-Gregory A, Hofmeyer A. Teaching empathy and resilience to undergraduate nursing students: $A$ call to action in the context of Covid-19. Nurse Educ Today. 2020 nov;94:104524. http:// dx.doi.org/10.1016/j.nedt.2020.104524. PMid:32771262.

24. Monforte-Royo C, Fuster P. Coronials: nurses who graduated during the COVID-19 pandemic. Will they be better nurses? Nurse Educ Today. 2020 nov;94:104536. http://dx.doi.org/10.1016/j.nedt.2020.104536. PMid:32801065.

25. Tracy S, McPherson S. Navigating COVID-19 through an unfolding case study for undergraduate nursing students. J Nurs Educ. 2020 ago 1;59(8):475-6. http://dx.doi.org/10.3928/01484834-20200723-11. PMid:32757014.

26. Prasad N, Fernando S, Willey S, Davey K, Kent F, Malhotra A et al Online interprofessional simulation for undergraduate health professional students during the COVID-19 pandemic. J Interprof Care. 2020 setout;34(5):706-10. http://dx.doi.org/10.1080/13561820.2020.1811213. PMid:32917099. 